\title{
Atomically precise clusters of gold and silver: A new class of nonlinear optical nanomaterials
}

\author{
Rodolphe Antoine* \\ Institut lumière matière, UMR5306 Université Claude Bernard Lyon1-CNRS, Université de Lyon 69622 Villeurbanne cedex, France \\ *Corresponding Author. Email: rodolphe.antoine@univ-lyon1.fr \\ Received: 06 September 2018, Accepted: 12 September 2018, Published Online: 13 September 2018 \\ Citation Information: Rodolphe Antoine. Frontier Research Today 2018;1:1001 doi: 10.31716/frt.201801001 Cite in Other Styles
}

ABSTRACT: Functional ligand-protected metal cluster nanomaterials with increased two-photon absorption and two-photon excited emission might result in new technologies within the fields of bio-imaging applications. During this article, I review the two-photon absorption/emission properties of atomically precise ligand-protected silver and gold nanoclusters, coined "ligand-core" NLO-phores. This includes quantumchemical methodologies using time-dependent density theory. I will analyze physical phenomena and trends resulting in giant two-photon absorption/emission responses of a number of series of model nanoclusters. I will then focus my review on the effects of the relaxation pathways within the linear and nonlinear optical regime, similarly as innovative ways aiming at enhancing their two-photon emission responses.

Keywords: Thiolated gold nanoclusters; Gold; Silver; High-yield synthesis; Mass-spectrometry; Electronic properties; Optical properties; Non-linear optics; Multi-photon microscopy

\section{INTRODUCTION}

Recent developments in optical imaging techniques, especially the multi-photon excitation (MPE) technique that permits studies of biological interactions at a deep cellular level, has intended intensive analysis in developing multi-photon absorption fluorophores. Biological tissues are optically transparent in the near-infrared region. Fluorophores that may absorb light within the NIR region by multi-photon absorption become significantly helpful in bio-imaging. The MPE technique permits molecular imaging by using either exogenous markers or endogenous signals. Endogenous non-linear optics (NLO) signals are usually weak and frequently need improvements of the instruments detection threshold and the development of scientific innovative targets, like endogenous fluorescent proteins ${ }^{1}$ to be applicable to MPE microscopy. Likewise, exogenous markers like organic dyes are synthesized for MPE research however their use remains restricted by their comparatively low two-photon excited visible radiation cross sections in liquid environments and their fast photo-bleaching. ${ }^{2}$ In addition, alternative probes are possible such as quantum dots with broad absorption and separate, tunable emission wavelengths withstanding photo-bleaching ${ }^{3}$. Preliminary studies have shown that gold or silver nanoclusters (Au or Ag NCs), nanomaterial fabricated from few to hundred gold or silver protected by thiolated molecules exhibiting molecular-like properties ${ }^{4}$, could also provide a valuable route in the nonlinear optical regime and MPE in particular.

Multi-photon optics stems from the nonlinear light-matter interaction, induced by an intense optical electric field $\mathrm{E}$ and is described by a medium polarization $\mathrm{P}=\chi^{(1)} \mathrm{E}+\chi^{(2)}$ $\mathrm{EE}+\chi^{(3)} \mathrm{EEE}+\ldots$, where $\chi^{(1)}$ is the linear susceptibility tensor representing effects such as linear absorption and refraction, $\chi^{(2)}$ is the second-order nonlinear optical susceptibility, $\chi^{(3)}$ is the third-order nonlinear susceptibility and so on. Sec- ond Harmonic Generation (SHG) is a second-order process whereas Two-Photon Excitation Fluorescence (TPEF) and Third Harmonic Generation (THG) are both third-order processes. The combination of the three processes intrinsically provides different contrasts for living matter microscopy (see Figure 1, left) ${ }^{5}$. Of note, the use of THG in the second NIR region $(1-1.4 \mu \mathrm{m}$ and $1.5-1.7 \mu \mathrm{m})$ is one of the strategy of choice for non-linear imaging in scattering tissue. $^{6}$

Designing highly efficient second $\left(\chi^{(2)}\right)$ and third order $\left(\chi^{(3)}\right)$ NLO chromophores is basically a matter of finely combining a high density of delocalized electrons in a symmetrical or unsymmetrical environment. Gold and silver NCs constitute therefore smart candidates (see Figure 1, right panel), though the reported $\left(\chi^{(2)}\right)$ efficiencies are still weaker than those of push-pull dyes ${ }^{7}$ and are not currently competitive as contrast agents for MPE applications. Our investigation on ligand protected silver and gold clusters ${ }^{8-15}$ has shown that the structure of the metal atom core, its charge and symmetry, dramatically influence the NLO cross-sections and the core stabilizing ligands play a key role in NLO efficiencies. We coined this new class of NLO materials : "Ligand-Core" NLO-phores ${ }^{16,17}$.

This feature article overviews mainly methodologies used for addressing the two-photon absorption (TPA) and two-photon excited (TPEF) emission properties of atomically precise clusters of gold and silver. In particular I will describe some strategies that have been suggested recently to enhance TPEF properties. This includes metal core-doping $^{18}$, a promising strategy as it distorts the metallic atom core, as well as strategies able to rigidify the protective shell ${ }^{19}$. Using results obtained on atomically precise clusters of silver, we get an extensive comprehension of the physics underlying the two-photon process and its amplitude, as well as to suggest an exploratory root for novel molecular engineering for further enhancement of TPA ${ }^{20}$. 


Two-photon
fluorescence (2PF)

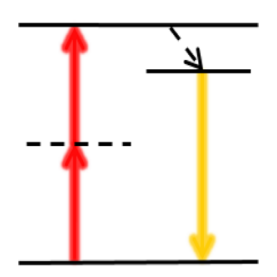

Second harmonic generation (SHG)

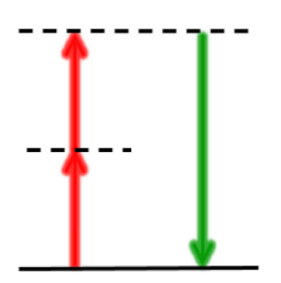

Third harmonic

generation (THG)

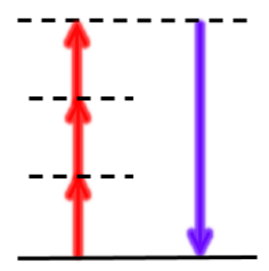

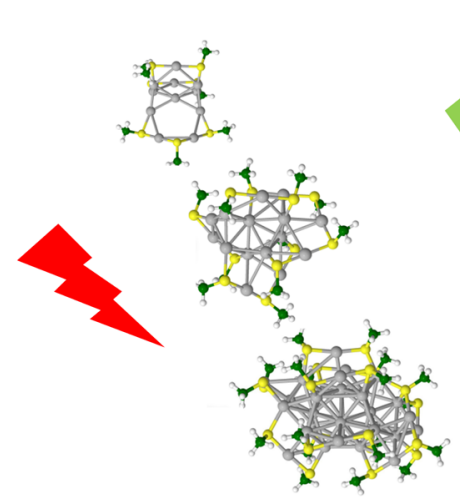

Figure 1. (left) Non-linear optics (NLO) processes: $2 \mathrm{PF}$ are used to image fluorescent dyes or endogenous molecules. SHG is used to image non-centrosymmetric structures such as collagen fibers and THG is used to visualize the refractive index differences such as the interface of lipid droplets. (right) Selected protected metal clusters that may display different NLO signals.

\section{SILVER: SYNTHESIS, CHARACTERIZATION AND OPTICAL PROPERTIES}

\subsection{Atomically precise clusters of gold and silver}

For small noble metal clusters -in the size range where each atom counts-, Mie-Drude-like model, that predicts the optical response of free-electron metals in the bulk state, is no longer appropriate to discuss absorption spectra in the details. The geometry of the clusters must be determined by quantum chemistry methods that often use group theory and the optic response are described in terms of molecular transitions whose positions and intensities are predicted by sophisticated calculations of quantum mechanics (vide infra). As a pioneering work, the absorption spectra obtained with the EOM-CCSD and STEOM-CCSD methods for the most stable structures of $\mathrm{Ag}_{5-8}$ nicely illustrated the molecular-like behavior of nanoclusters leading to an electronic energy quantization and the changes in the leading features of the patterns as a function of the cluster size $\mathrm{e}^{21}$. While the optical properties of such small metal clusters have been largely investigated in the gas phase, their study in the solid and liquid phase requires some "stabilization" that prevent them from fragmentation or degradation. The use of solid gas or inorganic matrices permits to protect gold or silver clusters from photodissociation ${ }^{22-25}$. Also, the large excited state charge separation is also a possible process in the photophysics of metals clusters composed by few atoms. Organic scaffolds allow the formation and stabilization of metal clusters in solution. The use of organic scaffolds for fluorescent metal nanoclusters are relatively new, first reported by the group of Dickson in 2002 for silver nanocluster $^{26}$. These organic scaffolds have tremendous potentials, as the interaction between the ligands and metal clusters can be adjusted leading to tunability in their spectroscopic properties. For example by using DNA oligomers as organic scaffolds and by playing with the nucleotide sequence of DNA oligomers it is possible to synthesize silver nanoclusters that emit from the blue to near-infrared region ${ }^{27}$.

Ligands play very important roles in the formation of NCs as protective agents, which can prevent the metal clusters from aggregation and then keep the size-dependent fluorescence property. The formation and stabilization of gold or silver nanoclusters in solution have been accomplished in various ways (see Figure 2). The proper choice of parameters for the reaction, including the temperature, the reducing method, the stabilizers and the initial ratio of metal salt:stabilizer, plays a crucial role for the successful synthesis of nanoclusters and to limit the size to few-atom nanoclusters. In addition to the ultrasmall size, the ligands used for NCs preparation also have impacts on their fluorescence properties. Jin and coworkers ${ }^{28}$ demonstrated that for gold NCs, the surface ligands of NCs not only can be used as capping agent but also largely affect the fluorescence of NCs through charge transfer from surface ligand to the gold core. When the surface ligands have strong electron donation capability, the fluorescence can be enhanced. And the ligands with electron-rich atoms or groups have been found as a very effective choice for promising surface ligand of NCs to enhance the fluorescence.

\subsection{Atomically precise clusters of gold and silver: synthetic routes}

The production of silver and gold nanoclusters can be performed following several routes. The metal ions from dissolved metal salts can be reduced, either by a chemical reductant (e.g. sodium borohydride, ...), (see Figure 3) by light (photoreduction with near-ultraviolet light) or by $\gamma$-rays (by radiolysis of water). The chemical reduction and the photoreduction are the most commonly used methods ${ }^{29}$. The specific properties of metal nanoclusters, such as the composition, stability and fluorescence quantum yield, depend largely on the scaffold used during reduction.

Thiols are frequently used on noble metal substrates because of the strong affinity of sulfur for these metals. And thiolated ligands (-SR) have appeared to be extremely good candidates to produce ultrasmall nanocluster sizes, in particular for gold ${ }^{30}$. Following the pioneering work of Brust et al. based on the reduction of the metal precursors and the formation of metal core, thiol-containing small molecules were extensively used to stabilize gold and silver nanoclusters in the aqueous solution ${ }^{31,32}$. The use of thiol-containing small molecules as stabilizers permits to better control the production of AuNCs than phosphine-capped ones, con- 


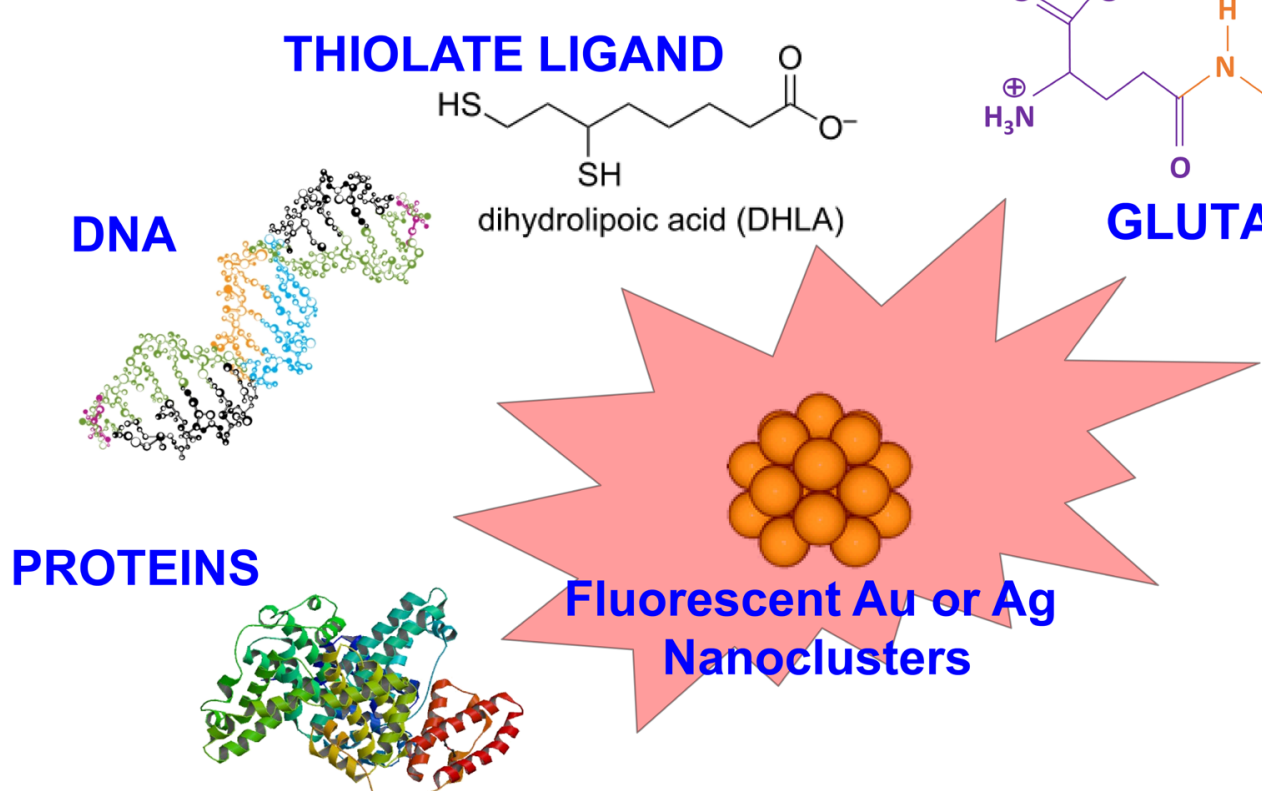

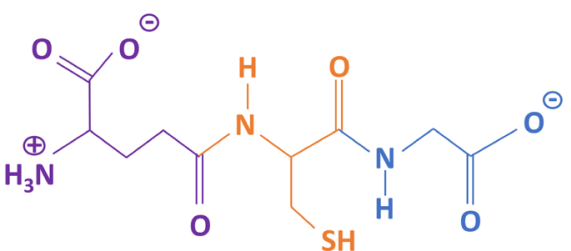

GLUTATHIONE

Figure 2. Protected metal nanoclusters with possible scaffolds.

tributing to the stronger Au-S covalent bonding. Generally, the method of synthesizing thiolate-capped AuNCs has processes as follows. Gold salts $\left[\mathrm{AuCl}_{4}\right]$ are dissolved in water and then transferred to an organic solvent by phase transfer agent; the thiols are added to the mixture induc- ing reduction of $\mathrm{Au}^{3+}$ ions into $\mathrm{Au}^{+}$ions and form $\mathrm{Au}^{+}-\mathrm{SR}^{-}$ complexes or polymers; then the $\mathrm{Au}^{+}$polymers are reduced by adding the reducing agent and lead to thiolate-protective gold nanoclusters.

Glutathione (GSH), a ubiquitous low-molecular weight
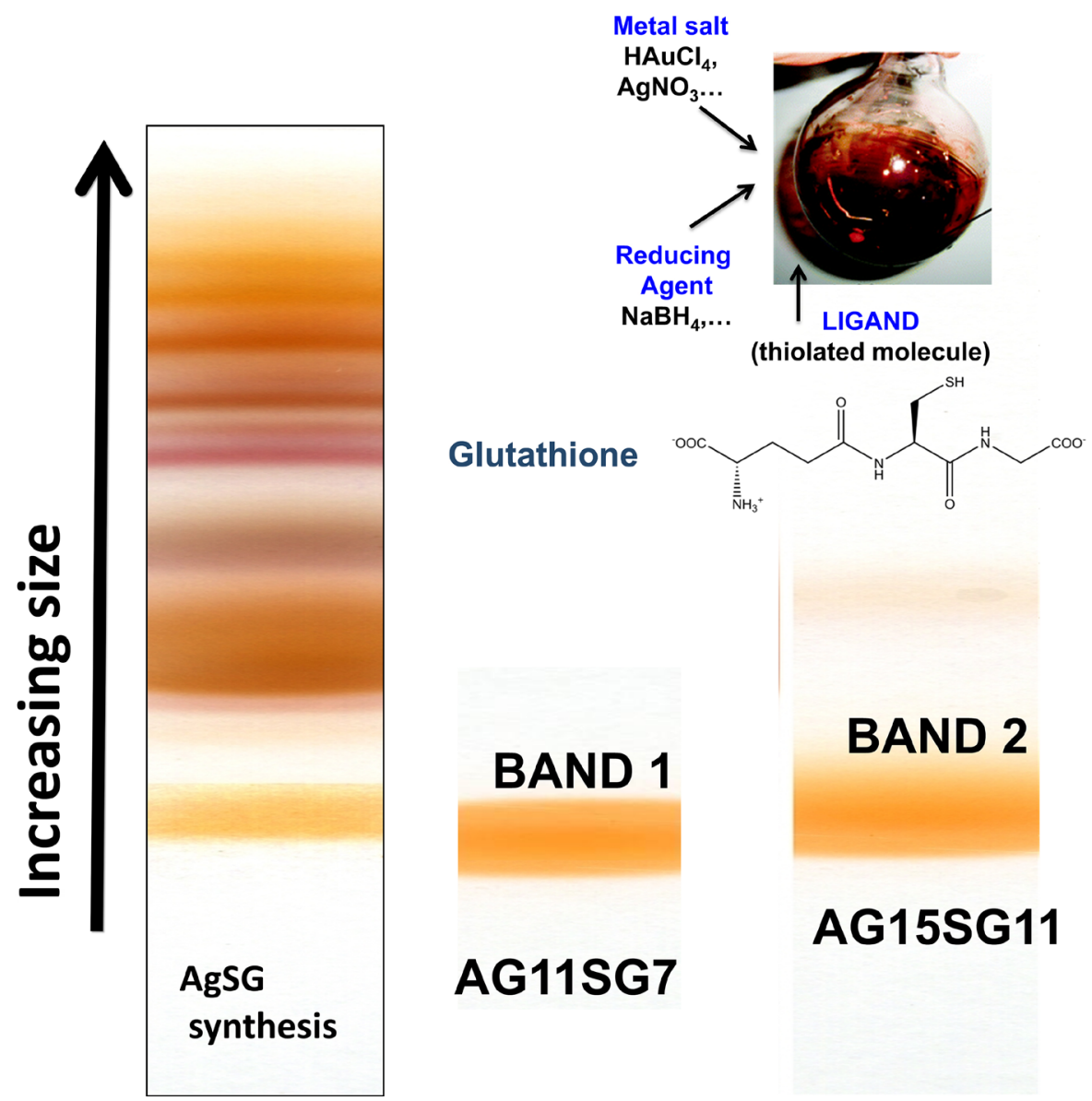

\section{BAND 6}
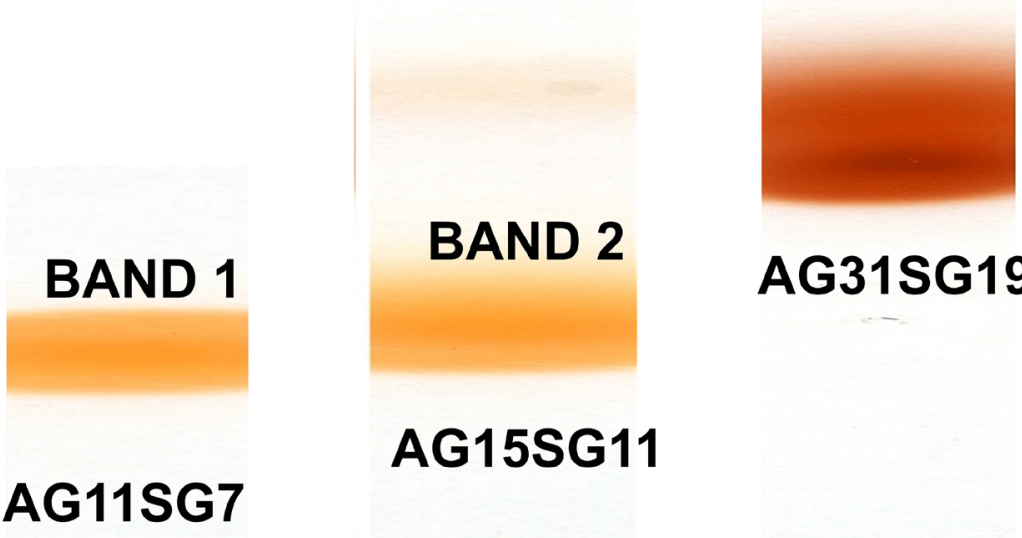

\section{BAND 2}

AG31SG19

Figure 3. (top panel) General route to produce thiolate protected nanoclusters by a chemical reductant.(bottom panel) PAGE for Ag:SG clusters by (left) Bigioni synthesis (see ref. 43) and (right) our focused Ag:SG synthesis. 
thiol, played a significant role in producing gold NCs which showed good water solubility, bioactive surface, and high stability. Whetten and coworkers have proposed an unprecedented thiol-protective AuNC route by using the GSH (N- $\gamma$-glutamyl-cysteinyl-glycine) as the stabilizer. The as-synthesized AuNCs were fractionated by using polyacrylamide gel electrophoresis (PAGE) and characterized by mass spectrometry (MS) ${ }^{33}$. Tsukuda and colleagues have also reported the characterization of fractionated AuNCs protected by GSH monolayers. The as-prepared AuNCs were isolated into single-sized $\mathrm{Au}_{\mathrm{n}}(\mathrm{SR})_{\mathrm{m}}$ clusters by the PAGE method and characterized using electrospray mass spectrometry $^{34,35}$.

While the routes for producing metal NCs lead to a mixture of $\mathrm{Au}_{\mathrm{n}}(\mathrm{SR})_{\mathrm{m}}$ cluster size, achieving atomic precision and molecular purity is challenging because the nanocluster growth is extremely complicated and remains poorly understood. Nevertheless, a systematic methodology called "size focusing", for achieving atomically precise clusters of gold and silver with molecular purity has been proposed. This methodology consists of two primary steps, (i) kinetically controlled synthesis of an $\mathrm{M}_{\mathrm{n}}(\mathrm{SR})_{\mathrm{m}}$ mixture with a properly controlled size range and (ii) thermodynamically dictated size-focusing of the mixture to single-sized nanoclusters. In parallel, a new approach, which is to utilize ligand exchange to induce size and structure transformation and, hence, to attain new $\mathrm{M}_{\mathrm{n}}(\mathrm{SR})_{\mathrm{m}}$ nanoclusters was also proposed $^{36}$.

Concerning the characterization techniques, X-ray crystallography is the "holy-grail" technique to solve the crystal structure of nanoclusters and reveals the nature of bonding and the packing of atoms ${ }^{4,37-39}$. Recently, complete structures have been experimentally resolved for many gold and silver nanoclusters, including the most popular $\mathrm{Au}_{25}(\mathrm{SR})_{18}$, $\mathrm{Au}_{38}(\mathrm{SR})_{24}$, and $\mathrm{Au}_{102}(\mathrm{SR})_{44} \mathrm{NCs}$ by X-ray crystallography ${ }^{4}$. Many other methods are used to characterize Au-NCs. X-ray photoelectron spectroscopy (XPS) is a valuable tool for confirming the oxidation state of gold in the sample, based on the $\mathrm{Au}-\mathrm{Au}$ and $\mathrm{Au}-\mathrm{S}$ binding energies. Various imaging techniques are widely employed to determine nanoparticle size and sample dispersity, such as transmission electron microscopy (TEM) and atomic force microscopy (AFM). Au $\mathrm{NC}$ composition is best resolved by high resolution mass spectrometry (MS). The most widely used ionization methods for Au NC characterization by MS are matrix-assisted laser desorption ionization (MALDI) and electrospray ionization (ESI) ${ }^{40-42}$.

\subsection{Atomically precise clusters of gold and silver: Optical properties}

One-photon excited fluorescence (OPEF) of metal nanoparticles is of considerable interest due to their potential applications in biomedicine for instance. However, the origin and underlying mechanism of OPEF in these clusters is still poorly understood, although some recent theoretical investigations by Christine M. Aikens, using time-dependent density functional theory (TD-DFT), shed some new lights on the key process for photon emission ${ }^{44-46}$. Unravelling the crystal structure has to better evaluate the structure-absorption relationships of some thiolate protected gold clusters. Time-dependent density functional theory (TDDFT) calculations on the electronic structure of $\mathrm{Au}_{25}$ clusters (using the reported structure with the single-crystal X-ray crystallographic analysis) have shown that the HOMO and the lowest three LUMOs are mainly composed of 6sp atomic orbitals of gold and a certain degree of the S (3p $)^{47}$. The other higher HOMO orbitals are mainly constructed from the $5 \mathrm{~d}^{10}$ atomic orbitals of gold and hence constitute the d-band. The calculated absorption transitions are in agreement with the experimental observations. Recent ultrafast spectroscopic studies on relaxation of higher excited states have provided more insights into understanding the photoluminescence mechanism of $\mathrm{Au}_{25}$ clusters. Goodson et al. found that $500 \mathrm{~nm}$ emission fundamentally arises from the electron-hole recombination in the $\mathrm{Au}_{13}$ core with little perturbation from surface ligands, but NIR emission at $700 \mathrm{~nm}$ originates from the recombination of holes in the ground core state and electron decay from core excited states to $\mathrm{S}-\mathrm{Au}-\mathrm{S}-\mathrm{Au}-\mathrm{S}$ semi rings ${ }^{48}$. Jin et al. recently found that charge state and surface ligands also have a significant influence on the NIR emission wavelength and QYs of $\mathrm{Au}_{25}$ clusters $^{49}$. Clearly, more detailed photophysical studies are required for evaluating how surface ligands influence the photoluminescence mechanisms of such systems. The last ten years, some general trends have been figured out concerning the de-excitation pathways following a visible or near-UV absorption. The following experimental and theoretical findings were assembled from the present work and literature to derive the energy diagram in Figure $4^{50}$.

Near-ultraviolet and visible absorbance may arise from transitions between molecular orbitals with high ligand contribution to orbitals with high metal character (LMCT) and from metal-metal electronic transitions. A rapid $(<1$ ps lifetime) decay pathway for clusters which have a core of metal atoms, that may lead to an emission in the visible. A long-lived (>100 ns lifetime), charge-transfer component is exhibited for all clusters. NIR emission in the clusters is related to the surface states and originates from the charge transfer excited state (see Figure 4).

\section{ATOMICALLY PRECISE CLUSTERS OF GOLD AND SILVER AS NEW NLO CHRO- MOPHORES: BACKGROUND AND DESIGN}

The understanding of the structure-property relationship of molecular TPA is of great importance for the rational design of optimized two-photon chromophores. We will use the analogy with push-pull molecules in order to describe how theoretical data can be used to get an extensive comprehension of the physics underlying the two-photon process and its amplitude, as well as to suggest an exploratory root for novel chemical engineering for further enhancement of TPA in atomically precise clusters of silver and gold.

Push-pull dipolar molecules are characterized by a low-lying, high-intensity absorption band, related to the intramolecular charge transfer (ICT) between the electron donor (D) and acceptor (A) groups (see Figure 5).

The TPA cross section of such molecules is considered to be governed basically by two factors: transition dipole moments and transition energies of the molecule. As seen 
below, the theoretical expression of TPA cross section $\left(\delta_{\mathrm{T}}\right.$ $\left.{ }_{\mathrm{PA}}(\omega)\right)$ based on the perturbation expansion is comprised of the numerator including transition dipole moments and the denominator including the transition energies and the incident photon energy. The energy term governs the wavelength dispersion of $\delta_{\mathrm{TPA}}(\omega)$ whereas the dipole moment term governs the overall magnitude of $\delta_{\mathrm{TPA}}(\omega)$.Thus, the structure-property relationship for the molecules with large $d_{\text {TPA }}(\omega)$ can be reduced by optimizing the transition dipole moments and frequencies involved in the TPA process.

It is commonly accepted that from the perturbation theory, the TPA cross section at the laser frequency of $\omega$ is given $\mathrm{as}^{51}$

$$
\delta_{T P A}(\omega)=\frac{(2 \pi)^{2} \omega^{2}}{(c h)^{2}} g(\omega)\left|S_{e g}\right|^{2}
$$

where $\mathrm{c}$ and $\mathrm{h}$ are the speed of light and the Planck constant, respectively. $g(\omega)$ denotes the normalized lineshape function of the TPA transition, $\left|\mathrm{S}_{\mathrm{eg}}\right|$ is so-called two-photon tensor.

The TPA cross section $\delta_{\mathrm{TPA}}(\omega)$ can be written in SI units as: ${ }^{.2}$

$$
\delta_{T P A}(\omega)=\frac{1}{5 c^{2} \hbar n^{2} \varepsilon_{0}^{2}} \frac{(\hbar \omega)^{2}}{\left(\hbar \omega_{e g}-\hbar \omega\right)^{2}+\Gamma_{e g}^{2}} \times\left[\frac{\left|\mu_{e g}\right|^{2}|\Delta \mu|^{2} \Gamma_{e g}}{\left(\hbar \omega_{e g}-2 \hbar \omega\right)^{2}+\Gamma_{e g}^{2}}+\frac{\left|\mu_{e g}\right|^{2}\left|\mu_{e e^{\prime}}\right|^{2} \Gamma_{e^{\prime} g}}{\left(\hbar \omega_{e^{\prime} g}-2 \hbar \omega\right)^{2}+\Gamma_{e^{\prime} g}^{2}}\right]
$$

The main parameters $\delta_{\text {TPA }}(\omega)$ responsible for are: change in the permanent dipole moment $\Delta \mu$; transition dipole moments $\mu_{\mathrm{eg}}, \mu_{\mathrm{ee}}$; angles between dipole moments; linewidth $\Gamma$, and detuning energies from intermediate and final states, $\left(\hbar \omega_{\mathrm{eg}}-\hbar \omega\right)$ and $\left(\hbar_{\omega e^{\prime} \mathrm{g}}-2 \hbar \omega\right)$.

Enhancement of $\delta_{\mathrm{TPA}}(\omega)$ can be obtained by playing with the following factors :

1. Increasing the transition dipole moments. In molecular design, this can be realized by increasing the $\pi$-conjugation length, or by introducing electron donor/acceptor groups. In non-centrosymmetric molecules, increasing the difference of the ground and excited state permanent dipole moments can also increase $\delta_{\mathrm{TPA}}(\omega)$.

2. Maximizing resonance terms. Decreasing the detuning energy between intermediate and ground states can significantly enhance $\delta_{\text {TPA }}(\omega)$. If the intermediate state is located halfway between ground state and final state, a "double resonance" condition can be achieved, which can lead to a dramatic enhancement of $\delta_{\mathrm{TPA}}(\omega)$.

3. Reducing the linewidth of the lowest energy one-photon transition.

Such factors have been figured out in details for design strategies and structure-property relations of cyanine and cyanine-like molecular structures with the goal of enhancing TPA in the near-IR for multiphoton fluorescence sensing applications ${ }^{52}$.

Atomically precise nanoclusters of silver or gold can be viewed as a "multi-shell system" composed by (1) a metallic core, (2) a metal-ligand interface, in particular with staple motifs leading to metal-sulfur bonds, and (3) the surface ligand molecules. These three shells may communicate in two different ways: charge transfer from ligand to metal core (analogy with ligand-to-metal charge transfer (LMCT) or ligand-to metal-metal charge transfer (LMMCT) observed in metal complexes) and through direct bonding or direct donation of delocalized electrons of electron-rich groups of the ligands ${ }^{17}$. Such "communications" between ligands and metal core may increase the transition dipole moments leading to enhanced $\delta_{\text {TPA }}(\omega)$.

Density functional theory (DFT) and its time-dependent version (TDDFT) have been used for determination of the structural and optical properties of ligand-protected silver and gold clusters (see Figure 6). There are two approaches for addressing two-photon absorption within analytic response method: First, the calculation of third-order frequency-dependent response function from the second hyperpolarizability in which imaginary part is related to TPA cross section; the second approach involves the single residue of the second-order response function or the first hyperpolarizability. The latter represents more practical way of computing the TPA cross section. In spite of the success, it is necessary to notice that in standard response theory, the response functions can diverge, since the response function has poles whenever one or more of the optical frequencies equal an excitation energy. This can lead to non-

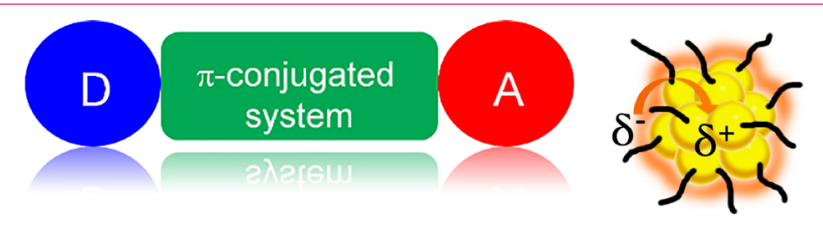

Figure 5. Schematic illustration of (left) a nonlinear optical (NLO), push-pull chromophore and (right) a "ligand-core" NLO-phore with ligand-protected silver and gold clusters. 

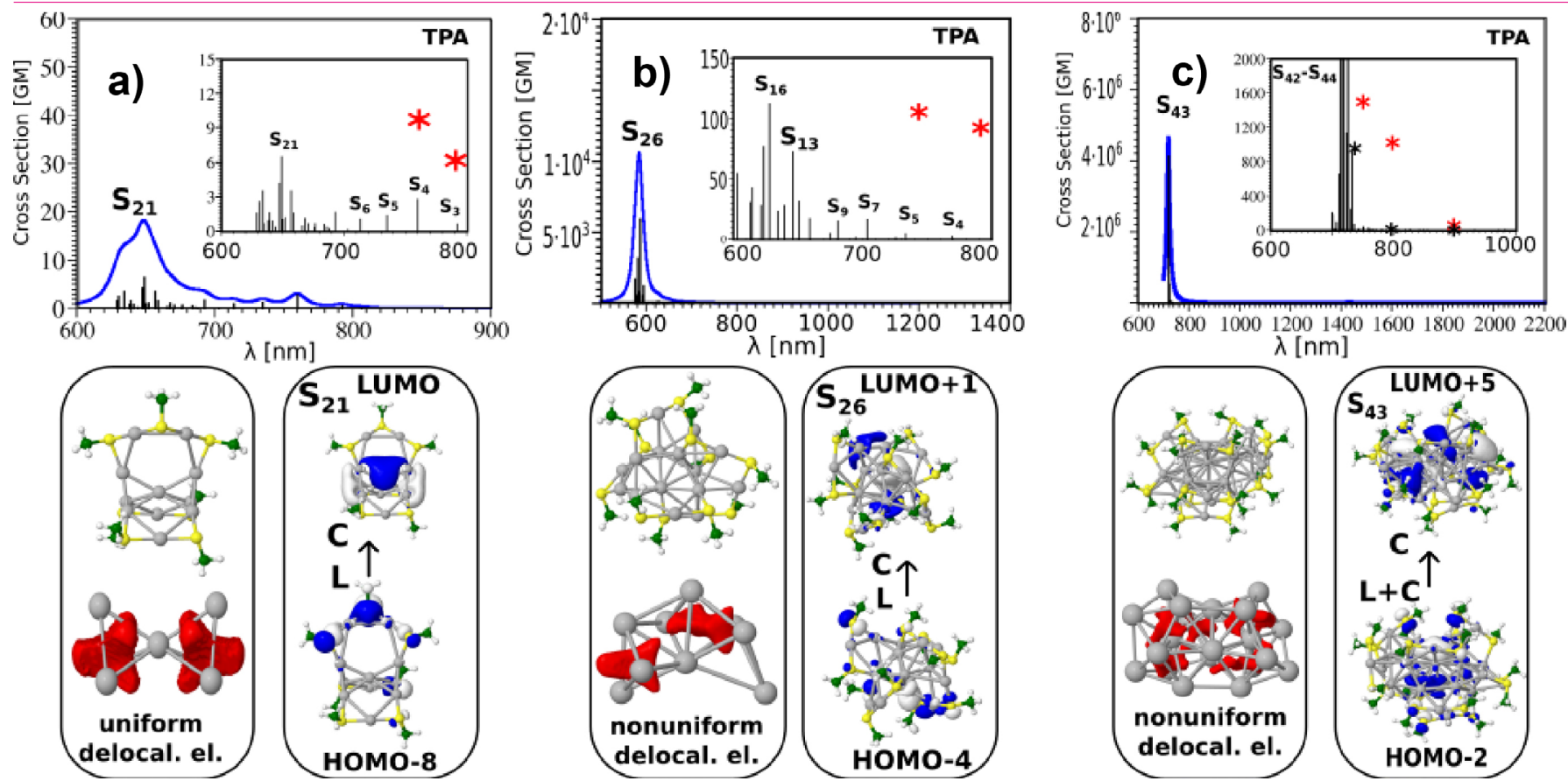

Figure 6. TDDFT TPA spectra of a) $\mathrm{Ag}_{11}\left(\mathrm{SCH}_{3}\right)_{7}$, b) $\mathrm{Ag}_{15}\left(\mathrm{SCH}_{3}\right)_{11}$ and c) $\mathrm{Ag}_{31}\left(\mathrm{SCH}_{3}\right)_{19}$ nanoclusters for the lowest energy structures involving 4,8 and 12 delocalized electrons in the metal core.

physical behavior for molecular properties in the resonance region. However, introducing damping terms in different ways the singularities of the response functions can be corrected or effectively removed ${ }^{14}$.

The two-photon absorption cross section for an excitation from the ground state $\mid 0>$ to a final state $|f\rangle$ is defined in terms of normalized shape function $\mathrm{g}\left(\omega_{\mu}+\omega_{\nu}\right)$ and the two-photon absorption transition amplitude tensor $\mathrm{T}^{(\omega \mu \omega \nu, \mathrm{f}}$

$$
\sigma_{T P A}=\frac{(2 \pi e)^{4} \omega_{\nu} \omega_{\mu}}{c^{2}} g\left(\omega_{\nu}+\omega_{\mu}\right)\left|T^{\omega_{\nu} \omega_{\mu}, f}\right|^{2}
$$

In order to obtain this tensor, the quadratic density functional response theory was applied. Two-photon absorption transition amplitude tensor $\left|T^{\omega_{\nu} \omega_{\mu}, f}\right|^{2}$ between the ground and the excited state is defined as

$$
T_{\text {b }}^{2 \omega, f}=\sum_{k}\left[\frac{\left\langle 0\left|\hat{\mu}_{a}\right| k\right\rangle\left\langle k\left|\hat{\mu}_{b}\right| f\right\rangle}{\omega_{k}-\omega_{f} / 2}+\frac{\left\langle 0\left|\hat{\mu}_{b}\right| k\right\rangle\left\langle k\left|\hat{\mu}_{a}\right| f\right\rangle}{\omega_{k}-\omega_{f} / 2}\right]
$$

where it is assumed that frequency of incident radiation is equal to half of excitation energy from ground to excited state, i.e. $\omega=\omega_{\mathrm{f}} / 2$. In the above equation $\mu_{\mathrm{a}}$ and $\mu_{\mathrm{b}}$ are Cartesian components of dipole moment operator $\mu$, and $\omega_{\mathrm{k}}$ and $\omega_{\mathrm{f}}$ are the frequencies of excitation from $\mid 0>$ to $\mid \mathrm{k}>$ and $\mid \mathrm{f}>$ respectively. Thus application of this formula includes explicit summation over excited states and requires computation of dipole moment operator $\mu$ between excited states. Of note, in order to prevent the TPA cross sections from blowing up near the one-photon resonances, the SOSs approach uses a damping factor $\Gamma$. More details about computational details can be found in ref. 14, 17 .

We conducted a theoretical investigation of the nonlinear optical properties for the lowest energy structures of the $\mathrm{Ag}_{11} \mathrm{~L}_{7}, \mathrm{AG}_{15} \mathrm{~L}_{11}$ and $\mathrm{Ag}_{31} \mathrm{~L}_{19}$ nanoclusters, where $\mathrm{L}$ stands for the $\mathrm{SCH}_{3}$ group (Figure 6) ${ }^{16}$. They contain respectively 4,8 and 12 delocalized electrons within the core. Several factors influencing the TPA cross-sections have been figure out: (i) the excitation between ligands and the metal core are characteristic of the nonlinear transitions, (ii) the "double resonance" between states involved in the OPA and TPA processes is required to obtain giant TPA cross-sections, (iii) large dipole transition moments are related to a non-uniform electron distribution within the metal core. The role of the structural properties, i.e. of the geometry of the metal core in determining this electron distribution, is therefore crucial.

In the case of the TPA cross-sections, interplay between a resonance effect and large transition dipole moments is essential. As the size of the silver nanoclusters increases, transition dipole moments should continue to increase due to larger core size (and thus larger core-to-ligand distances) and/or non-uniform electronic distribution in the metal core. Also, the optical band gap decreases as the size of the nanoclusters increases ${ }^{39}$, shifting the spectra towards the NIR spectral region, a feature useful for bio-imaging applications.

\section{4 "LIGAND-CORE" NLO-PHORES}

\subsection{NLO emission of $\mathrm{Ag}_{29}(\mathrm{DHLA})_{12}$. Playing with photons and colors}

A general observation: these NCs have huge two-photon absorption cross sections typically 50,000 $\mathrm{GM}^{\# 1}$ for $\mathrm{Ag}_{29}$ against their cross section for two-photon emission is only $0.5 \mathrm{GM}^{13}$. For NLO applications, they are still less efficient than dyes but they are biocompatible and present low toxicity unlike dyes. We have also observed for the $\mathrm{Ag}_{29}$ an interesting behavior for potential applications, which concerns its emission properties following the absorption of one or two photons, as shown in Figure 7. With a photon at $400 \mathrm{~nm}$ or 2 photons at $800 \mathrm{~nm}$, an emission spectrum of either blue or red is obtained. So we have one single nanoobject with two colors, using linear or nonlinear optics. This 

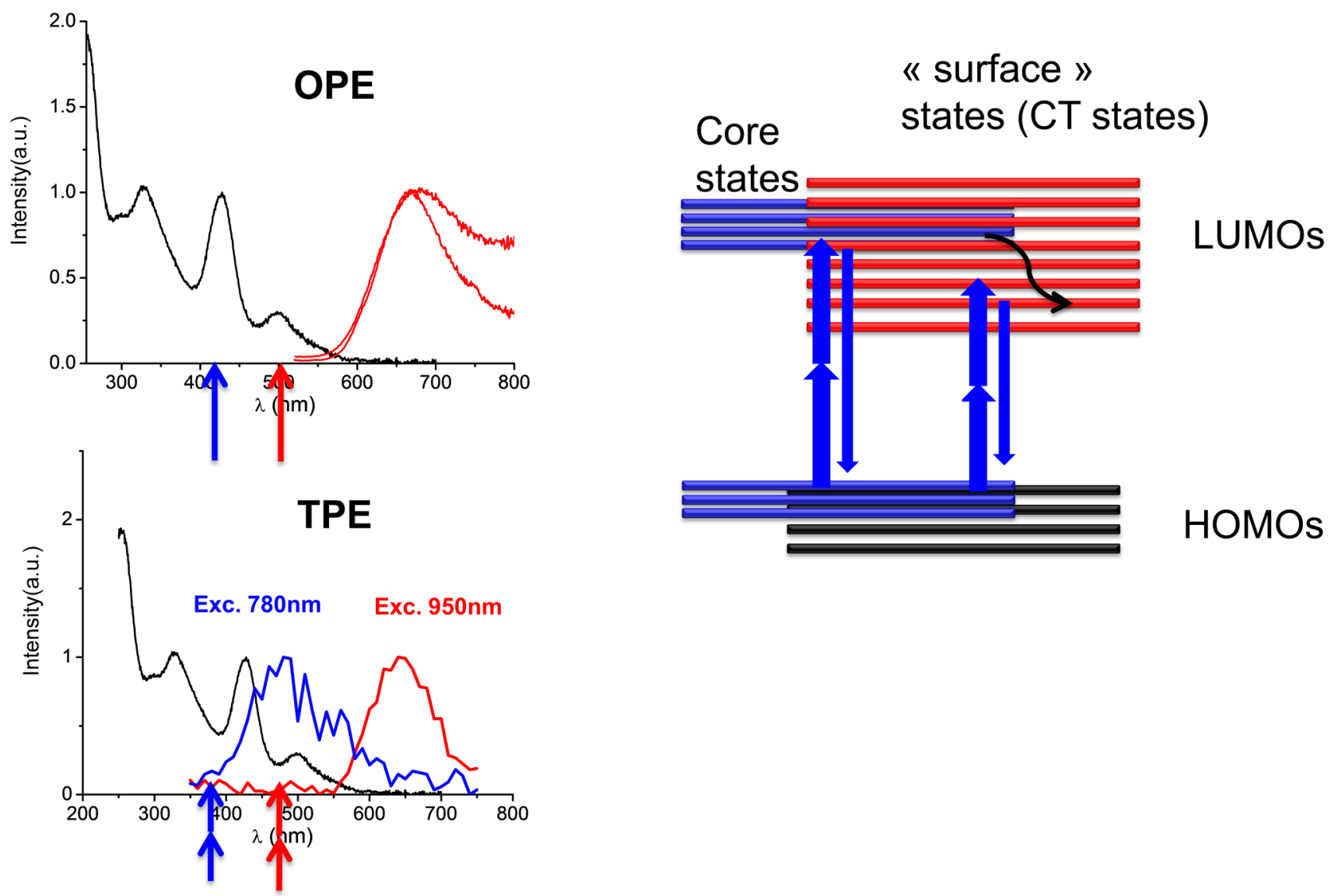

Figure 7. (left panel) Spectroscopic characterization of the synthesized $\operatorname{Ag}_{29}(\text { DHLA) })_{12}$ clusters dispersed in water. Absorption spectrum (black), OPEF spectrum (top) and TPEF spectrum (bottom). The arrows represent the wavelength of excitation by OPE and TPE processes (right panel) Schematics showing the proposed excited state relaxation dynamics in the $\mathrm{Ag}_{29}(\text { DHLA })_{12}$ clusters in TPEF experiments.

behavior is still poorly understood and probably reflects the complexity of the relaxation of excited electronic states characteristic of the molecular or quantum regime.

For organic molecules, the emission typically occurs from its lowest excited energy level for a given spin multiplicity (referred to as Kasha's rule). However nanoclusters as well as certain organic molecules tend to break this rule and emit at more than one possible wavelength depending on the electronic structure, density of states and excited state dynamics involved. Regardless of whether a molecule/ nanocluster obeys the Kasha's rule or not, in order to gain a more detailed understanding of the excited state emission dynamics of a chromophore, one may carry out the time resolved experiments.

Note that the emission spectra upon one- and two-photon excitation (Figure 4) are almost identical except for the region below $400 \mathrm{~nm}$, such deviations might be the "signature" of a deviation from the Kasha's rule... Note that the photochemistry of gold nanoclusters is more complex than S1, S2 $\pi-\pi^{*}$ states of organic molecules, where excitations within the gold core as well as couplings with surface states (through LMCT and LMMCT) may occur.

4.2 Bulky counterions. A simple route to enhance the TPEF efficiencies

At this stage, we reckon that protected gold quan- tum clusters are excellent two-photon absorbers but rather poor two-photon excited emitters ${ }^{53}$. To enhance emission efficiencies, the ligand shell rigidity is an interesting strategy that would allow for enhanced photon emission as compared to non-radiative relaxation upon photo-excitation. Pyo et al. ${ }^{19}$ have shown that it is possible to achieve luminescence quantum yield $>60 \%$ by rigidifying the metal-sulfur interface with the binding of bulky groups. It is possible to push forward these concepts, using either core-doping or rigidification, to the nonlinear optical regime.

The strategy developed recently is to use voluminous ammonium counter-ions, a nitrogen cation surrounded by four alkyl chains. In solution, the fluorescence yields of NCs are often affected by the solvent (in particular water), the counter-ions will come by electrostatic interaction (between the counterions and the carboxylates of the glutatione) to stick to the surface of the NCs which will have the effect of protecting it from the environment and also to "rigidify" its surface. The effect is spectacular on the one-photon fluorescence, as shown on $\mathrm{Au}_{15}$ in water, which then complexed with tetrabutylammonium becomes extremely fluorescent (see Figure 8). We extended this study to the NLO regime and were able to show that the same dramatic effect is observed for TPEF and with a variety of large counterions, the best candidate to date remains tetrabutylammonium (see Figure 8). The TPEF cross sections

${ }^{\# 1}$ Maria Goeppert-Mayer, Nobel Prize in Physics 1963. Her name is now known for the unit of the molecular TPA cross-section $(G M): 1$ GM $=10^{-50} \mathrm{~cm}^{4}$. s.photons ${ }^{-1}$. 

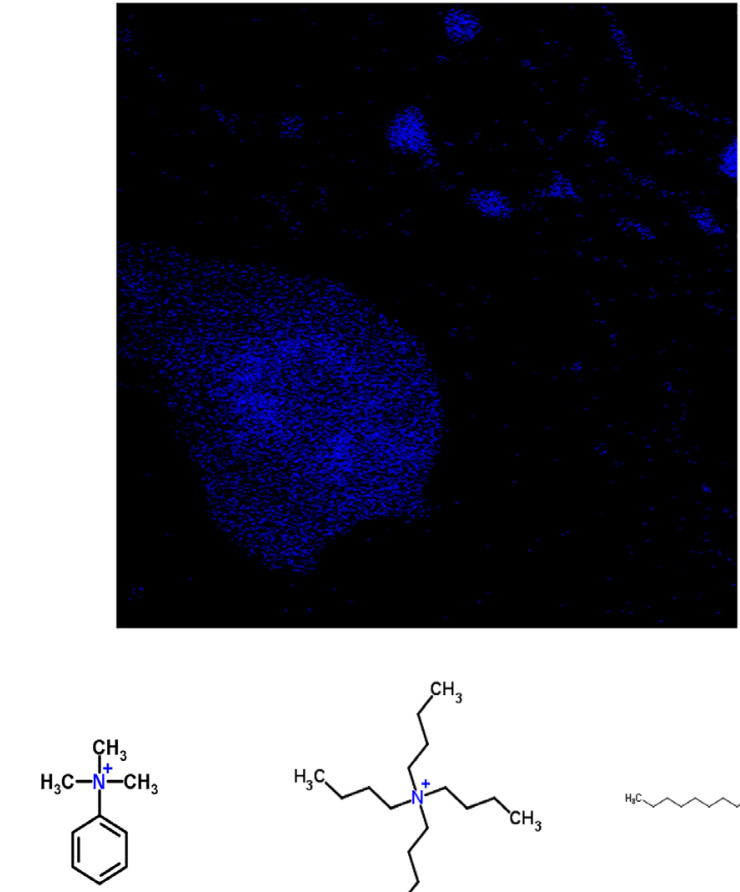

TMph : BzTMA

trimethylphenyl ammonium

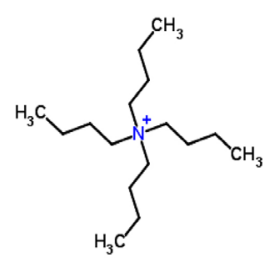

TBA :

Tetrabutyl ammonium

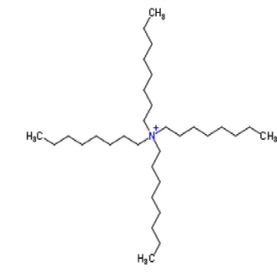

TOA :

Tetraoctyl ammonium
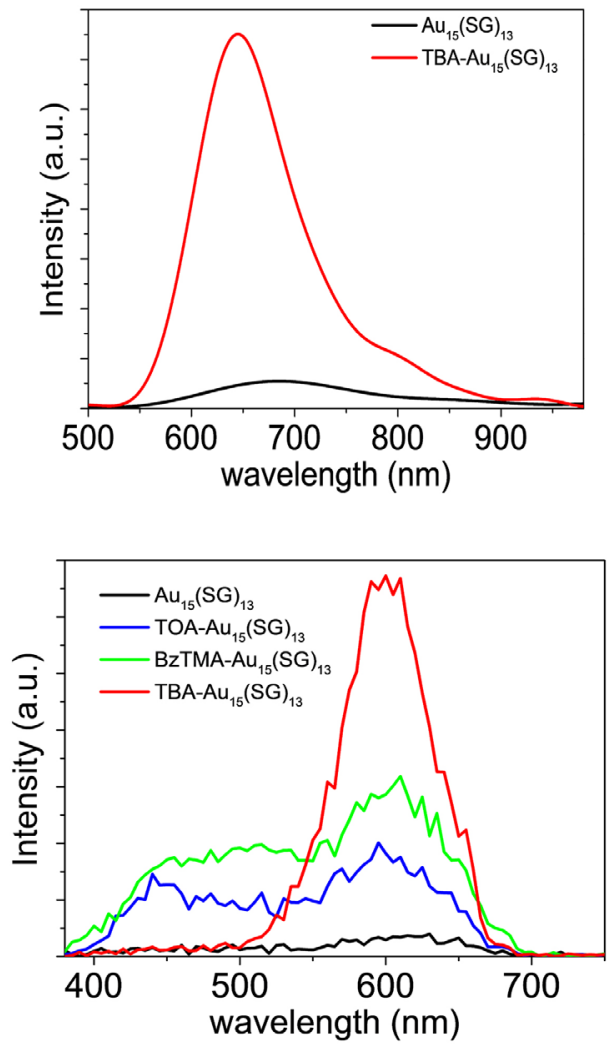

Figure 8. (left panels) (top) TPEF image of Au NCs containing methanol droplets in heptane and (bottom) structure of bulky counterions used to bind to glutathione-protected gold clusters. (right panels) (top) One-photon excited fluorescence spectra of $\mathrm{Au}_{15}(\mathrm{SG})_{13}$ in water and $\mathrm{TBA}-\mathrm{Au}_{15}(\mathrm{SG})_{13}$ in methanol. (bottom) Two-photon excited fluorescence spectra at excitation wavelength $780 \mathrm{~nm}$ of $\mathrm{Au}_{15}(\mathrm{SG})_{13}$ in aqueous solution compared to different bulky cations- $\mathrm{Au}_{15}(\mathrm{SG})_{13}$ in methanol.

of these objects then become interesting for multiphoton optics and we have crossed the road towards 2-photon confocal microscopy.

Factors $~ 30$ gained on TPEF cross-sections allowed confocal imaging under $780 \mathrm{~nm}$ excitation. As a proof of concept and before going to cell imaging, we made an emulsion from a water-heptane mixture, it gives rise to droplets of water trapped in heptane of micrometric size. NCs, in this case gold in the presence of counterions are soluble in water and were used to image the droplets. The image of TPEF in false blue color is shown in Figure 8, where we recognize the droplet containing the NCs. The pause time for stack images is only few seconds, which shows the efficiency for two-photon imaging.

\section{CONCLUDING REMARKS}

The aim of the present study was to gain fundamental knowledges on the mechanism involved in multiphoton processes in atomically precise gold and silver nanoclusters (NCs). By measuring two photon absorption, two photon excited fluorescence cross sections and first hyperpolarizability for such NCs, we have provided unique benchmarks for theoretical modeling of the origin of enhanced NLO properties of ultra-small ligated metal clusters, as well as the interplay between the cluster core and the interface between the ligand shell and the metallic part.

It will also serve as a basis for further developments in the design of high efficiency NLO-phores with good stability and low toxicity for in vitro (and possibly ultimately in vivo) bio-imaging applications. One of the technological objective of this study will be to bridge the gap between new cell biomarkers and two-photon NLO-phores design.

Designing highly efficient second- and third-order nonlinear optical (NLO) chromophores is largely a matter of finely combining a high density of delocalized electrons in a symmetrical or unsymmetrical environment. For enhancing NLO efficiencies, innovative strategies should be explored in the future. To enhance second order $\chi^{(2)}$ efficiencies, metal core-doping is a promising strategy as it distorts the metallic atom core ${ }^{54}$. Also, enhanced fluorescence of AuNCs by silver doping was reported by Le Guével et al. ${ }^{55,56}$ Therefore to enhance third order $\chi^{(3)}$ efficiencies, silver doping can be coupled to ligand shell rigidity as the latter favors the radiative properties of AuNCs. This route was recently explored by Olesiak-Banska et al. who showed that $\mathrm{Au} / \mathrm{Ag}$ NCs exhibit two-photon excited luminescence emission and second-harmonic generation (SHG) and that these properties remain the same in liquid crystalline matrix ${ }^{56}$. An alternative route to enhance TPEF efficiencies, is to use the concept of "rigidity". We recently pushed forward this strategy consisting in using bulky counterions to enhance the luminescence in the nonlinear optical regime. We showed that by an appropriate choice of bulky counterions and of solvent, a 30-fold increase in TPEF signal in the red for glutathione-protected gold clusters could be obtained. A simple way to increase rigidity of the protective shell is to replace thiolated ligands by proteins instead. Bovine serum albumin- $\mathrm{Au}_{25} \mathrm{NCs}$ indeed exhibit an efficient two-photon 
absorption followed by blue ${ }^{57}$ to red to near-infrared photoluminescence ${ }^{58}$. Using (bio)organic thiolate templates, further enhancement can be achieved by the increasing rigidity of the metal-sulfur interface. The precise tailoring of the hydrophilicity/hydrophobicity balance on the Au NC surfaces can be a way to increase the fluorescence signal ${ }^{59,60}$.

\section{Notes}

The authors declare no competing financial interest.

\section{Acknowledgements}

Most of the work presented would not have been possible without the fruitful collaborations with Isabelle Russier-Antoine, Franck Bertorelle, Željka Sanader, Marjan Krstić, Philippe Dugourd, Pierre-François Brevet and Vlasta Bonačić-Koutecký. Therefore, I wish to express my deepest gratitude to them. Furthermore, I would like to acknowledge financial support of the French-Croatian project "International Laboratory for Nano Clusters and Biological Aging, LIA NCBA".

\section{References}

[1] De Meulenaere E, Nguyen Bich N, de Wergifosse M, Van Hecke K, Van Meervelt L, Vanderleyden J, et al. Improving the Second-Order Nonlinear Optical Response of Fluorescent Proteins: The Symmetry Argument. Journal of the American Chemical Society 2013;135(10):4061-69. doi:10.1021/ja400098b

[2] Terenziani F, Katan C, Badaeva E, Tretiak S, Blanchard-Desce M. Enhanced Two-Photon Absorption of Organic Chromophores: Theoretical and Experimental Assessments. Advanced Materials 2008;20(24):4641-78. doi:10.1002/adma.200800402

[3] Medintz IL, Uyeda HT, Goldman ER, Mattoussi H. Quantum dot bioconjugates for imaging, labelling and sensing. Nature Materials 2005;4:435. doi:10.1038/nmat1390

[4] Jin R, Zeng C, Zhou M, Chen Y. Atomically Precise Colloidal Metal Nanoclusters and Nanoparticles: Fundamentals and Opportunities. Chemical Reviews 2016;116(18):10346-413. doi:10.1021/ acs.chemrev.5b00703

[5] Tu H, Liu Y, Marjanovic M, Chaney EJ, You S, Zhao Y, et al. Concurrence of extracellular vesicle enrichment and metabolic switch visualized label-free in the tumor microenvironment. Science Advances 2017;3(1):e1600675. doi:10.1126/sciadv.1600675

[6] Yue S, Slipchenko MN, Cheng JX. Multimodal nonlinear optical microscopy. Laser \& Photonics Reviews 2011;5(4):496-512. doi:10.1002/lpor.201000027

[7] Blanchard-Desce M. Molecular engineering of NLO-phores for new NLO microscopies. Comptes Rendus Physique 2002;3(4):43948. doi:10.1016/S1631-0705(02)01329-4

[8] Bellina B, Antoine R, Broyer M, Gell L, Sanader Z, Mitric R, et al. Formation and characterization of thioglycolic acid-silver cluster complexes. Dalton Transactions 2013;42(23):8328-33. doi:10.1039/c3dt50485a

[9] Bellina B, Compagnon I, Bertorelle F, Broyer M, Antoine R, Dugourd P, et al. Structural and Optical Properties of Isolated Noble Metal-Glutathione Complexes: Insight into the Chemistry of Liganded Nanoclusters. Journal of Physical Chemistry C 2011;115:24549-54. doi:10.1021/jp207158v

[10] Bertorelle F, Hamouda R, Rayane D, Broyer M, Antoine R, Dugourd P, et al. Synthesis, characterization and optical properties of low nuclearity liganded silver clusters: Ag31(SG)19 and Ag15(SG)11. Nanoscale 2013;5(12):5637-43. doi:10.1039/ c3nr00677h

[11] Bertorelle F, Russier-Antoine I, Calin N, Comby-Zerbino C, Bensalah-Ledoux A, Guy S, et al. $\mathrm{Au}_{10}(\mathrm{SG})_{10}$ : A Chiral Gold Catenane Nanocluster with Zero Confined Electrons. Optical Properties and First-Principles Theoretical Analysis. The Journal of Physical Chemistry Letters 2017;8(9):1979-85. doi:10.1021/acs. jpclett.7b00611

[12] Bonacic-Koutecky V, Kulesza A, Gell L, Mitric R, Antoine R, Bertorelle F, et al. Silver cluster-biomolecule hybrids: from basics towards sensors / perspective article. Physical Chemistry Chemical Physics 2012;14:9282-90. doi:10.1039/c2cp00050d

[13] Russier-Antoine I, Bertorelle F, Hamouda R, Rayane D, Dugourd P, Sanader Z, et al. Tuning Ag29 nanocluster light emission from red to blue with one and two-photon excitation. Nanoscale 2016;8(5):2892-98. doi:10.1039/C5NR08122J

[14] Sanader Z, Krstic M, Russier-Antoine I, Bertorelle F, Dugourd $\mathrm{P}$, Brevet P-F, et al. Two-photon absorption of ligand-protected $\mathrm{Ag}_{15}$ nanoclusters. Towards a new class of nonlinear optics nanomaterials. Physical Chemistry Chemical Physics 2016;18:1240408. doi:10.1039/C6CP00207B

[15] Sanader Z, Mitric R, Bonacic-Koutecky V, Bellina B, Antoine R, Dugourd P. Nature of electronic excitations at the metal-bioorganic interface illustrated on histidine-silver hybrids. Physical Chemistry Chemical Physics 2014;16(3):1257-61. doi:10.1039/ c3cp52712c

[16] Russier-Antoine I, Bertorelle F, Calin N, Sanader Z, Krstic M, Comby-Zerbino C, et al. Ligand-core NLO-phores: a combined experimental and theoretical approach to the two-photon absorption and two-photon excited emission properties of small-ligated silver nanoclusters. Nanoscale 2017;9(3):1221-28. doi:10.1039/ C6NR07989J

[17] Antoine R, Bonacic-Koutecky V. Liganded silver and gold quantum clusters. Towards a new class of nonlinear optical nanomaterials. Materials Si, editor: Springer, Cham 2018.

[18] Jin R, Nobusada K. Doping and alloying in atomically precise gold nanoparticles. Nano Research 2014;7(3):285-300. doi:10.1007/ s12274-014-0403-5

[19] Pyo K, Thanthirige VD, Kwak K, Pandurangan P, Ramakrishna G, Lee D. Ultrabright Luminescence from Gold Nanoclusters: Rigidifying the $\mathrm{Au}(\mathrm{I})-$ Thiolate Shell. Journal of the American Chemical Society 2015;137(25):8244-50. doi:10.1021/jacs.5b04210 [20] Bonačić-Koutecký V. Theoretical design of new class of optical materials based on small noble metal nanocluster-biomolecule hybrids and its potential for medical applications. Advances in Physics: X 2017;2(3):695-716. doi: 10.1080/23746149.2017.1352458 [21] Bonačić-Koutecky V, Veyret V, Mitrić R. Ab initio study of the absorption spectra of Agn ( $n=5-8)$ clusters. The Journal of Chemical Physics 2001;115(22):10450-60. doi:10.1063/1.1415077

[22] Fedrigo S, Harbich W, Buttet J. Collective dipole oscillations in small silver clusters embedded in rare-gas matrices. Physical Review B 1993;47(16):10706-15. doi:10.1103/PhysRevB.47.10706

[23] Harbich W, Fedrigo S, Buttet J. The Optical-Absorption Spectra of Small Silver Clusters (N = 5-11) Embedded in Argon Matrices. Chem Phys Lett 1992;195(5-6):613-17. doi:10.1016/00092614(92)85572-R

[24] Harbich W, Fedrigo S, Buttet J, Lindsay DM. Optical spectroscopy on size selected gold clusters deposited in rare gas matrices. Zeitschrift für Physik D Atoms, Molecules and Clusters 1991;19(4):157-59. doi:10.1007/BF01448280

[25] Harbich W, Fedrigo S, Meyer F, Lindsay DM, Lignieres J, Rivoal JC, et al. Deposition of mass selected silver clusters in rare gas matrices. The Journal of Chemical Physics 1990;93(12):8535- 
43. doi:10.1063/1.459291

[26] Zheng J, Dickson RM. Individual Water-Soluble Dendrimer-Encapsulated Silver Nanodot Fluorescence. Journal of the American Chemical Society 2002;124(47):13982-83. doi:10.1021/ ja0282821

[27] Richards CI, Choi S, Hsiang J-C, Antoku Y, Vosch T, Bongiorno A, et al. Oligonucleotide-Stabilized Ag Nanocluster Fluorophores. Journal of the American Chemical Society 2008;130(15):5038-39. doi:10.1021/ja8005644

[28] Wu Z, Jin R. On the Ligand's Role in the Fluorescence of Gold Nanoclusters. Nano Letters 2010;10(7):2568-73. doi:10.1021/ nl101225f

[29] Diez I, Ras RHA. Fluorescent silver nanoclusters. Nanoscale 2011;3(5):1963-70. doi:10.1039/c1nr00006c

[30] Jin R. Quantum sized, thiolate-protected gold nanoclusters. Nanoscale 2010;2(3):343-62. doi:10.1039/B9NR00160C

[31] Jin R, Zeng C, Zhou M, Chen Y. Atomically Precise Colloidal Metal Nanoclusters and Nanoparticles: Fundamentals and Opportunities. Chemical Reviews 2016;116:10346-413. doi:10.1021/acs. chemrev.5b00703

[32] Udayabhaskararao T, Pradeep T. New Protocols for the Synthesis of Stable Ag and Au Nanocluster Molecules. J Phys Chem Lett 2013;4:1553-64. doi:10.1021/jz400332g

[33] Schaaff TG, Knight G, Shafigullin MN, Borkman RF, Whetten RL. Isolation and selected properties of a $10.4 \mathrm{kDa}$ Gold : Glutathione cluster compound. The Jorunal of Physical chemistry B 1998;102(52):10643-46. doi:10.1021/jp9830528

[34] Negishi Y, Nobusada K, Tsukuda T. Glutathione-protected gold clusters revisited: Bridging the gap between gold(I)-thiolate complexes and thiolate-protected gold nanocrystals. J Am Chem Soc 2005;127(14):5261-70. doi:10.1021/ja042218h

[35] Negishi Y, Takasugi Y, Sato S, Yao H, Kimura K, Tsukuda T. Magic-numbered Au-n clusters protected by glutathione monolayers $(n=18,21,25,28,32,39)$ : Isolation and spectroscopic characterization. Journal of the American Chemical Society 2004;126(21):6518-19. doi:10.1021/ja0483589

[36] Zeng C, Chen Y, Das A, Jin R. Transformation Chemistry of Gold Nanoclusters: From One Stable Size to Another. The Journal of Physical Chemistry Letters 2015;6(15):2976-86. doi:10.1021/acs. jpclett.5b01150

[37] Qian H, Eckenhoff WT, Zhu Y, Pintauer T, Jin R. Total Structure Determination of Thiolate-Protected Au-38 Nanoparticles. Journal of the American Chemical Society.132(24):8280-1. doi:10.1021/ja103592z

[38] Zhu M, Aikens CM, Hollander FJ, Schatz GC, Jin R. Correlating the Crystal Structure of A Thiol-Protected Au25 Cluster and Optical Properties. Journal of the American Chemical Society 2008;130(18):5883-85. doi:10.1021/ja801173r

[39] Jin R. Atomically precise metal nanoclusters: stable sizes and optical properties. Nanoscale 2015;7(5):1549-65. doi:10.1039/ C4NR05794E

[40] Hamouda R, Bertorelle F, Rayane D, Antoine R, Broyer M, Dugourd P. Glutathione capped gold AuN(SG)M clusters studied by isotope-resolved mass spectrometry. International Journal of Mass Spectrometry 2013;335:1-6. doi:10.1016/j.ijms.2012.10.008

[41] Chakraborty I, Pradeep T. Atomically Precise Clusters of Noble Metals: Emerging Link between Atoms and Nanoparticles. Chemical Reviews 2017;117(12):8208-71. doi:10.1021/acs.chemrev.6b00769

[42] Lu Y, Chen W. Application of Mass Spectrometry in the Synthesis and Characterization of Metal Nanoclusters. Analytical Chemistry 2015;87(21):10659-67. doi:10.1021/acs.anal- chem.5b00848

[43] Kumar S, Bolan MD, Bigioni TP. Glutathione-Stabilized Magic-Number Silver Cluster Compounds. Journal of the American Chemical Society 2010;132(38):13141-43. doi:10.1021/ja105836b

[44] Weerawardene KLDM, Aikens CM. Origin of Photoluminescence of Ag25(SR)18- Nanoparticles: Ligand and Doping Effect. The Journal of Physical Chemistry C 2018;122(4):2440-47. doi:10.1021/acs.jpcc.7b11706

[45] Weerawardene KLDM, Guidez EB, Aikens CM. Photoluminescence Origin of Au38(SR)24 and Au22(SR)18 Nanoparticles: A Theoretical Perspective. The Journal of Physical Chemistry C 2017;121(28):15416-23. doi:10.1021/acs.jpcc.7b01958

[46] Weerawardene KLDM, Aikens CM. Theoretical Insights into the Origin of Photoluminescence of Au25(SR)18- Nanoparticles. Journal of the American Chemical Society 2016;138(35):11202-10. doi:10.1021/jacs.6b05293

[47] Fernando A, Weerawardene KLDM, Karimova NV, Aikens CM. Quantum Mechanical Studies of Large Metal, Metal Oxide, and Metal Chalcogenide Nanoparticles and Clusters. Chemical Reviews 2015;115(12):6112-216. doi:10.1021/cr500506r

[48] Devadas MS, Kim J, Sinn E, Lee D, Goodson T, Ramakrishna G. Unique Ultrafast Visible Luminescence in Monolayer-Protected Au25 Clusters. The Journal of Physical Chemistry C 2010;114(51):22417-23. doi:10.1021/jp107033n

[49] Wu ZK, Jin RC. On the Ligand's Role in the Fluorescence of Gold Nanoclusters. Nano Lett 2010;10(7):2568-73. doi:10.1021/ nl101225f

[50] Zheng J, Zhou C, Yu M, Liu J. Different sized luminescent gold nanoparticles. Nanoscale 2012;4(14):4073-83. doi:10.1039/ c2nr31192e

[51] Birge RR, Pierce BM. A theoretical analysis of the twophoton properties of linear polyenes and the visual chromophores. The Journal of Chemical Physics 1979;70(1):165-78. doi:10.1063/1.437217

[52] Przhonska OV, Webster S, Padilha LA, Hu H, Kachkovski AD, Hagan DJ, et al. Two-Photon Absorption in Near-IR Conjugated Molecules: Design Strategy and Structure-Property Relations. In: Demchenko AP, editor. Advanced Fluorescence Reporters in Chemistry and Biology I: Fundamentals and Molecular Design. Berlin, Heidelberg: Springer Berlin Heidelberg 2010. p. 105-47.

[53] Russier-Antoine I, Bertorelle F, Vojkovic M, Rayane D, Salmon E, Jonin C, et al. Non-linear optical properties of gold quantum clusters. The smaller the better. Nanoscale 2014;6(22):13572-78. doi:10.1039/C4NR03782K

[54] Van Steerteghem N, Van Cleuvenbergen S, Deckers S, Kumara C, Dass A, Häkkinen H, et al. Symmetry breaking in ligand-protected gold clusters probed by nonlinear optics. Nanoscale 2016;8(24):12123-27. doi:10.1039/C6NR02251K

[55] Le Guevel X, Trouillet V, Spies C, Li K, Laaksonen T, Auerbach $\mathrm{D}$, et al. High photostability and enhanced fluorescence of gold nanoclusters by silver doping. Nanoscale 2012;4(24):7624-31. doi:10.1039/c2nr30653k

[56] Brach K, Waszkielewicz M, Olesiak-Banska J, Samoc M, Matczyszyn K. Two-Photon Imaging of 3D Organization of Bimetallic AuAg Nanoclusters in DNA Matrix. Langmuir 2017;33(36):8993-99. doi:10.1021/acs.langmuir.7b00873

[57] Kindi HA, Mohamed A, Kajimoto S, Zhanpeisov N, Horino $\mathrm{H}$, Shibata Y, et al. Single bovine serum albumin molecule can hold plural blue-emissive gold nanoclusters: A quantitative study with two-photon excitation. Journal of Photochemistry and Photobiology A: Chemistry 2018;357:168-174. doi:10.1016/j.jphotochem.2018.02.029 
[58] Raut SL, Shumilov D, Chib R, Rich R, Gryczynski Z, Gryczynski I. Two photon induced luminescence of BSA protected gold clusters. Chemical Physics Letters 2013;561(Supplement C):74-6. doi:10.1016/j.cplett.2013.01.028

[59] Porret E, Sancey L, Martín-Serrano A, Montañez MI, Seeman R, Yahia-Ammar A, et al. Hydrophobicity of Gold Nanoclusters Influences Their Interactions with Biological Barriers. Chemistry of Materials 2017;29(17):7497-506. doi:10.1021/acs.chemmater.7b02497

[60] Shen D, Henry M, Trouillet V, Comby-Zerbino C, Bertorelle F, Sancey L, et al. Zwitterion functionalized gold nanoclusters for multimodal near infrared fluorescence and photoacoustic imaging. APL Materials 2017;5(5):053404. doi:10.1063/1.4977203

\section{Open Access}

This article is licensed under a Creative Commons Attribution 4.0 International License.

(c) The Author(s) 2018 